\title{
The Health Canada Surveillance Tool could be an effective method for assessing alignment with 2019 Canada's Food Guide
}

\author{
Christine Mulligan, Beatriz Franco-Arellano and Mary R L'Abbe \\ University of Toronto, Toronto, Canada
}

\section{Abstract}

The Health Canada Surveillance Tool (HCST), a Canadian nutrient profile (NP) model, assesses products' adherence to the 2007 Canada's Food Guide (CFG), using thresholds for total fat, saturated fat, sugars and sodium. In 2019, new dietary guidelines were published (i.e., CFG 2019); however; the HCST has not been updated to reflect changes implemented in this new guide. Given suggestions to adapt previously validated NP models rather than create new models, this research aimed to assess whether the HCST could be a useful tool to assess alignment with updated dietary guidance. Specifically, the objective of this study was to test the agreement between products' alignment with the CFG 2007 (as per the HCST) and products' alignment with the recently released CFG 2019 guidelines. This study analyzed data from the University of Toronto Food Label Information Program (FLIP) 2017 database. FLIP contains label and nutrition information for prepackaged food products from top Canadian grocery retailers. Products were categorized into Tiers based on HCST thresholds: Tiers 1 and 2 were considered "in line" with dietary guidance, while Tiers 3, 4 and "Other" (i.e. foods not addressed by CFG) were considered "not in line". Two raters independently classified foods according to their alignment to CFG 2019. Proportions of products that were considered "in line" with CFG 2007 and 2019 were calculated. Overall agreement between alignment with CFG 2007 and 2019 was determined by cross-classifications of the proportion of products considered "in line" or "not in line" with both CFG versions. Cohen's Kappa ( $\kappa$ ) statistic tested the level of agreement (Interpretation of $\kappa:$ 0.01-0.20, "slight"; 0.21-0.40, "fair"; 0.41-0.60, "moderate"; 0.61-0.80, "substantial"; and 0.81-0.99, "almost perfect"). Analyses were conducted overall and by Health Canada's Table of Reference Amounts for Food category. In total, $n=$ 16,973 products were analyzed, with $98 \%$ inter-rater reliability for CFG 2019 alignment. Overall, $30.2 \%$ and $28.2 \%$ of products were "in line" with CFG 2007 and 2019, respectively, with 80.4\% overall agreement and "moderate" kappa agreement ( $\kappa$ [95\% $\mathrm{CI}]: 0.49$ [0.46, 0.49]). Overall agreement in individual food categories ranged from 100\% (Dessert Toppings, Sauces, Sugars and Sweets; $\kappa:$ N/A) to $54.8 \%$ (Eggs, $\kappa: 0.21[-0.01,0.4]$ ). From these results, the HCST appears to be an effective NP model for assessing alignment with CFG 2019. Further analysis could elucidate specific areas for adaptation of the HCST to optimize its functionality in this context.

\section{Conflict of Interest}

C.M. completed a Mitacs graduate student internship at Nestlé Canada. Prior to coming to the University of Toronto, B.F.-A. was a PepsiCo Mexico employee (2009-2015). T.P. was a graduate student employed part-time by Intertek Scientific and Regulatory Consultancy. M.R.L. reports grants from the Retail Council of Canada; Program for Food Safety, Nutrition and Regulatory Affairs at the University of Toronto (with partial funding from Nestlé Canada); and Dairy Farmers of Canada; unrelated to the submitted work. None of these companies/organizations were involved in any way with regards to the present research. 